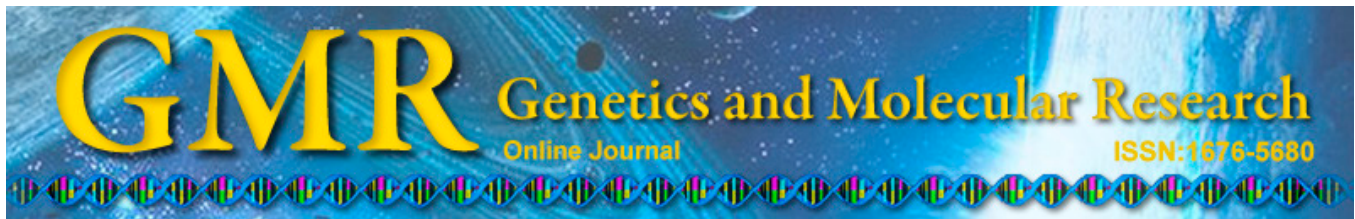

\title{
Molecular cloning and expression analysis of MyD88 in spiny head croaker, Collichthys lucidus
}

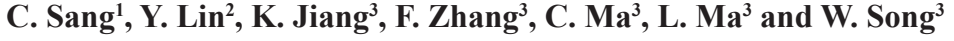 \\ ${ }^{1}$ School of Ocean, Yantai University, Yantai, Shangdong, China \\ ${ }^{2}$ College of Fisheries and Life Science, Shanghai Ocean University, \\ Shanghai, China \\ ${ }^{3}$ East China Sea Fisheries Research Institute, \\ Chinese Academy of Fishery Sciences, Shanghai, China
}

Corresponding author: W. Song

E-mail: songw@ecsf.ac.cn

Genet. Mol. Res. 14 (2): 4666-4676 (2015)

Received August 13, 2014

Accepted December 8, 2014

Published May 4, 2015

DOI http://dx.doi.org/10.4238/2015.May.4.26

\begin{abstract}
Myeloid differentiation factor 88 (MyD88) is an important adaptor protein involved in toll-like receptor signaling pathways. In this study, a cDNA library from Collichthys lucidus was constructed using the SMART technique. A complete cDNA sequence showing high identity with the conserved sequence of the MyD88 gene was cloned from the cDNA library using expressed sequence tag analysis and rapid amplification of cDNA ends, and then subjected to further investigation. The full-length cDNA of MyD88 from C. lucidus $(C l M y D 88)$ was $1580 \mathrm{bp}$, including a 5'-terminal untranslated region (UTR) of $102 \mathrm{bp}$, a 3'-terminal UTR of $614 \mathrm{bp}$, and an open reading frame of $864 \mathrm{bp}$. The gene encoded a polypeptide of 287 amino acids, constituting a predicted molecular weight of $33.03 \mathrm{kDa}$ and a theoretical isoelectric point of 5.06. It contained a typical death domain at the $\mathrm{N}$-terminal and a conservative toll/IL-1 receptor domain structure at the C-terminal. Quantitative real-time reverse transcription PCR analysis
\end{abstract}


revealed broad expression of $C l M y D 88$, with the highest expression in the gill and the weakest expression in the brain and muscle. These results indicated that MyD88 has an important role in the innate immune system in C. lucidus.

Key words: Collichthys lucidus; MyD88; Quantitative real-time PCR

\section{INTRODUCTION}

Like many other vertebrates, teleost fish have developed an innate and adaptive immune system to eliminate invading pathogens. Usually, innate immune responses are triggered upon pathogen recognition by a set of pattern recognition receptors (PRRs) that recognize conserved pathogen-associated molecular patterns (PAMPs) such as bacteria, fungi, and viruses, or sometimes even protozoa (Takeda and Akira, 2005). Toll-like receptors (TLRs) are the most famous PRRs (Armant and Fenton, 2002; Bilak et al., 2003; Beutler, 2005) and comprise a family of transmembrane receptors that can recognize various kinds of PAMPs, such as peptidoglycan, lipoproteins, double-stranded viral RNA, lipopolysaccharide and unmethylated bacterial $\mathrm{CpG}$ DNA (Hawlisch and Kohl, 2006). Six major TLRs have been identified in all vertebrate taxa, and are TLR1, TLR3, TLR4, TLR5, TLR7, and TLR11 based on phylogenetic analysis (Jared et al., 2005). Myeloid differentiation factor 88 (MyD88) is an important adaptor protein involved in the activation of interleukin-1 receptor (IL-1R) and nuclear factor-kappaB (NF-kB) by TLRs (Yao et al., 2009). In general, TLR signaling can be segregated into two distinct pathways: the MyD88-dependent and MyD88-independent pathways (Medzhitov et al., 1998). Studies have shown that the MyD88-dependent pathway is the most prominent, and is activated by all TLRs except TLR3 (Bonizzi and Karin, 2004). In the MyD88-dependent pathway, after the recognition of PAMPs by TLRs, MyD88 is the first molecule recruited to the activated TLR through homophilic interactions between the TIR (TLR and IL-1R-related) domains of the receptor and MyD88. On the other hand, the death domain of MyD88 associates with the death domain of IL-1R-associated kinase (IRAK) (Muzio et al., 1997; Wesche et al., 1997). Next, the signaling cascade is initiated to activate NF-kB and mitogen-activated protein kinases (Burns et al., 1998; Medzhitov et al., 1998; Janssens and Beyaert, 2002). Finally, the transcription of a multitude of immune effectors that are involved in the response against invading pathogens is initiated (Qiu et al., 2007). MyD88 was also reported to be involved IL-1R-related signaling due to the cytoplasmic TIR domains (Janssens and Beyaert, 2002).

Spiny head croaker Collichthys lucidus is a small economically important sciaenid species that inhabits coastal waters of the northwestern Pacific ranging from Kyushu, Japan, to the South China Sea. As a migrator of short distances and a shallow sea fish, C. lucidus dominates quantitatively demersal fish catches off the coasts of China and Japan, supporting an important commercial fishery. Although C. lucidus is highly appreciated by Chinese consumers because of its excellent properties and quality of the meat, limited research of $C$. lucidus in recent years has mainly focused on the mitogenome, microsatellites, and auxology. So far no gene sequence has been cloned in C. lucidus. Although MyD88 plays a critical role in initiating and activating the immune system, little is known about this protein in this species. More information about MyD88 in other teleost fishes is needed to better understand the immune function of the TLR signaling pathway and its evolution.

In this study, we described the characterization of the full-length cDNA of MyD88 
in C. lucidus (ClMyD88), deduced its amino acid sequences, predicted the three-dimensional (3D) structure and relative expression profiling in different tissues, hoping to clarify its potential role in immune responses in C. lucidus.

\section{MATERIAL AND METHODS}

\section{Materials and reagents}

Healthy spiny head croaker $C$. lucidus averaging $20 \pm 5 \mathrm{~g}$ in weight were collected from Ningde, China. Different tissues including the gill, kidney, liver, brain, muscle, spleen, heart, and hemocyte were collected and immediately preserved in liquid nitrogen for RNA extraction. Hemocytes were withdrawn with a needle from the tail of each $C$. lucidus into a syringe containing pre-cooled $\left(-4^{\circ} \mathrm{C}\right)$ anticoagulant $(0.45 \mathrm{M} \mathrm{NaCl}, 0.1 \mathrm{M}$ glucose, $30 \mathrm{mM}$ trisodiumcitrate, $26 \mathrm{mM}$ citric acid, and $10 \mathrm{mM}$ EDTA, $\mathrm{pH}$ 4.6) to reach a volume ratio of 1:1. This was then pelleted by centrifugation $(8000 \mathrm{rpm})$ (AllegraTM64R Centrifuge, Beckman Coulter, USA) at $4^{\circ} \mathrm{C}$ for $10 \mathrm{~min}$.

\section{cDNA library construction}

A C. lucidus cDNA library was successfully constructed by using a SMART ${ }^{\mathrm{TM}}$ cDNA library construction kit (Clontech, Palo Alto, CA, USA) and all the EST sequences were subjected to BLAST analysis. An expressed sequence tag (EST) that was highly similar to that of a previously identified MyD88 sequence was selected for further cloning of ClMyD 88 cDNA.

\section{Total RNA extraction and first strand cDNA synthesis}

Total RNA was isolated from the gill using Unizol reagents (Biostar, Shanghai, China) following the protocol of the manufacturer, and then stored at $-80^{\circ} \mathrm{C}$. The quality and concentration was checked by agarose gel electrophoresis and spectrophotometry (DU 800, Beckman Coulter, Pasadena, CA, USA).

First-strand cDNA synthesis was performed using moloney murine leukemia virus reverse transcriptase (Promega Corporation, Madison, WI, USA) to transcribe poly(A) mRNA using Oligo-dT and random 6-mer primers. The reaction conditions were as recommended by the manufacturer.

\section{3'rapid amplification of cDNA ends (RACE) and 5'RACE}

The missing $5^{\prime}$ and $3^{\prime}$ ends of MYD88 cDNA of C. lucidus were obtained by RACE method, which was performed using the SMART ${ }^{\mathrm{TM}}$ RACE CDNA amplification kit (Clontech, Palo Alto, CA, USA) according to manufacturer instructions. Primers for $3^{\prime}$ and 5'-RACE were: MyD88-3race (5'-GATGCCTGCGACTTTCAGACCA-3') and MyD88-5race (5'-CGCAGGCATCACTGTCAAGGTA-3').

\section{Sequence analysis}

Identity searches for nucleotide and protein sequences were performed using the BLAST algorithm at NCBI (http://www.ncbi.nlm.nih.gov/). The deduced amino acid se- 
quence was analyzed with the expert protein analysis system (http://www.expasy.org/). Amino acid sequences from various species were retrieved from the NCBI GenBank and analyzed using the Vector NTI Suite 11.0, Clustal W multiple alignment program (http://www.ebi.ac.uk/ clustalw/). A neighbor-joining (NJ) phylogenetic tree was constructed using MEGA software version 4.1 and the confidence level in the generated tree was obtained using 1000 bootstraps. The secondary structure was predicted by the application of the hierarchical neural network. The 3D structure of $C l M y D 88$ was simulated using the SWISS-MODEL long-distance server.

\section{Quantitative real-time PCR (qRT-PCR) analysis of ClMyD88 in various tissues}

Total RNA was isolated from different tissues of C. lucidus using the TRIzol reagent (TaKaRa, Shiga, Japan) and then stored at $-80^{\circ} \mathrm{C}$. Total RNA $(1 \mu \mathrm{g})$ was reverse transcribed with the Rever Tra Ace qPCR RT kit (Toyobo Co., Ltd., Osaka, Japan) to generate first strand cDNA. The product was diluted $1: 10$ and stored at $-20^{\circ} \mathrm{C}$ until qRT-PCR analysis was performed. The process was as follows: the first strand cDNA was reverse transcribed using 500 ng total RNA from muscle by using 10-fold serial dilutions to generate the standard curve. The SYBR GreenI qRT-PCR assay [SYBR Premix Ex Taq ${ }^{\mathrm{TM}}$ Green (Perfect Real time), TaKaRa] was carried out in an ABI Step OnePlus Detection system (Applied Biosystems, Foster City, CA, USA). Amplifications were performed on a 96 -well plate with a $20-\mathrm{mL}$ reaction volume containing $10 \mathrm{~mL}$ SYBR Premix Taq ${ }^{\mathrm{TM}}, 0.8 \mathrm{~mL}$ PCR forward primer $(10 \mathrm{mM}), 0.8 \mathrm{~mL}$ PCR reverse primer $(10 \mathrm{mM}), 0.4$ $\mathrm{mL}$ ROX reference dyeI (503), $2.0 \mathrm{~mL}$ cDNA temple, and $6.0 \mathrm{~mL}$ diethylpyrocarbonate (DEPC) water. The thermal profile for SYBR Green qRT-PCR was as follows: $30 \mathrm{~s}$ at $95^{\circ} \mathrm{C}$, followed by 40 cycles at $95^{\circ} \mathrm{C}$ for $5 \mathrm{~s}$, and $60^{\circ} \mathrm{C}$ for $34 \mathrm{~s}$. DEPC-water was used to replace the template for a negative control. Fluorescence data were collected at completion of each cycle.

In this experiment, the preliminary trial showed that the housekeeping gene 18S rRNA had steady expression in this experimental species. Therefore, 18S rRNA was used as the internal housekeeping gene in all qRT-PCR assays, and 18s-RT-F (5'-GCC TGA ATA CCG CAG CTA GGAATA A-3') and 18s-RT-R (5'-TTT CAC CTC TAG CGG CAC AAT ACG-3') were designed based onthe 18S rRNA gene sequence (GenBank accession No. JN211725.1). The standard curve and levels of gene expression were analyzed automatically by the system, as was the setting of the base line. Melting curve analyses of amplified products were performed at the end of each PCR to confirm that only one product was amplified and detected. For the qRT-PCR results, the observations in different tissues were calculated to derive the means and standard deviations. Primers used to amplify MyD88 were MyD88-RT-F (5'-ACTACGAAGCGACCAATAACCC-3') and MyD88-RT-R (5'-CCGTCAGCACCGACAGCA-3').

\section{RESULTS AND DISCUSSION}

Molecular features and functions of genes have become a hotspot of recent research. Various functional genes have been cloned, investigated, and described in fish of Sciaenidae. For instance, many genes have been reported in Pseudosciaena crocea that have a very close relationship with C. lucidus, including TLR1 (Wang et al., 2013), TLR7 (Qian et al., 2013), TLR8 (Qian et al., 2013), and TLR22 (Xiao et al., 2011), which are believed to be involved in TLR signaling pathways. However, no gene sequence has been cloned from C. lucidus. Knowing that TLRs represent a primary line of defense against invading pathogens in mammals, and that MyD88 is a key adaptor for almost all TLRs (Bonizzi and Karin, 2004; Takeda and Akira, 
2005), we cloned the first full-length cDNA sequence of MyD88 from C. lucidus.

\section{Cloning of ClMyD88}

It was found in this study that the full-length cDNA fragment of $C l M y D 88$ consisted of $1580 \mathrm{bp}$ (GenBank accession No. KM247622), with open reading frames (ORF) of $864 \mathrm{bp}$; a 102-bp 5'-UTR, and a 614 bp 3'-UTR with a poly(A) tail, and that consensus polyadenylation signals (AATAAA) were present 11b pupstream of the poly(A) tails. Based on the deduced polypeptide sequence, the ORF should encode a putative protein of 287 amino acids with a predicted molecular weight of $33.03 \mathrm{kDa}$ and a theoretical isoelectric point of 5.06. In addition, two typical MyD88 domains (a death domain and a TIR domain) were identified in the $C l M y D 88$ deduced amino acid sequence by the SMART program. An N-glycosylation site was also found at position 20-23. The full-length nucleotide sequence and the deduced amino acid sequence are shown in Figure 1.

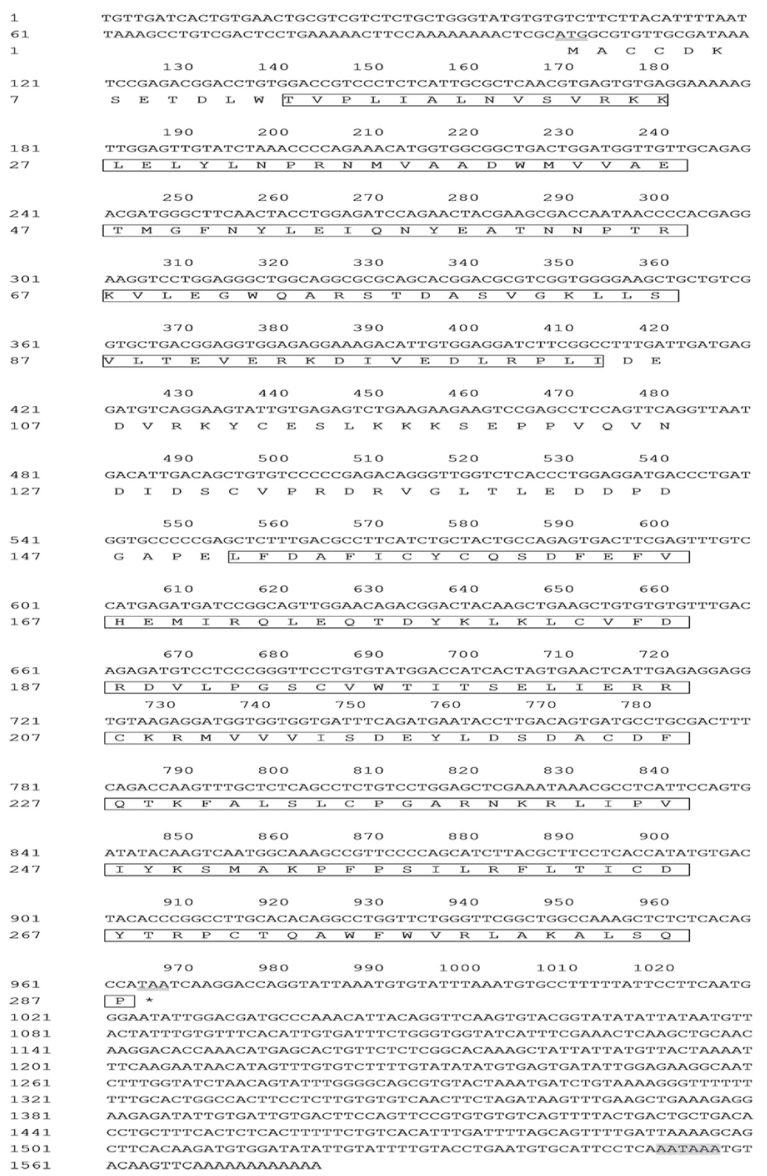

Figure 1. Nucleotide sequence and deduced amino-acid sequence of ClMyD 88 cDNA. The start (ATG) and stop (TAA) codons are underlined. The instability motif (AATAAA) is shaded. The amino acid sequence in box indicates the death domain that extends from 13 to 104 and the TIR domain that extends from 151 to 287. 
Some conserved domain residues were found in ClMyD88. An N-terminal death domain (DD domain) and a C-terminal TIR domain (TIR domain) were identified in the deduced ClMyD88 amino acid sequences at positions 13-104 and 151-287, respectively, which are similar to the typical domain structure of mammalian MyD88 and other fish (Takano et al., 2006; Qiu et al., 2007; Yao et al., 2009). This suggests that ClMyD88 may have a similar function in toll signaling transduction in this species. Previous research has reported that the death domain super family is a prime mediator of the interactions necessary for death signal transduction, regulation of apoptosis, and inflammatory responses (Itoh and Nagata, 1993; Tartaglia and Ayres, 1993; Weber and Vincenz, 2001; Takano et al., 2006). In mammals, MyD88 and IRAK suse their death domains for binding interactions (Wesche et al., 1997). While in Drosophila, DmMyd88 has a death domain to mediate interactions with Pelle and Tube (Tauszig-Delamasure et al., 2002). The TIR domain, which plays an important role in activating innate immune responses via the IL-1R/TLR super family mediated pathway (Janssens and Beyaert, 2003), was reported to be more highly conserved than DD domains (Takano et al., 2006). In mammals, the TIR domain is approximately 150 amino acids long and contains three conserved regions, boxes 1,2 , and 3, which play an important role in signal recognition and engaging with downstream elements (Tauszig-Delamasure et al., 2002). In addition, the three conserved functional boxes were also identified in ClMyD88 and other fishes, such as Japanese flounders (Takano et al., 2006) and large yellow croakers (Yao et al., 2009). This indicates that the TIR domains of fishes may have the same important role as mammalian MyD88in TLR signaling.

\section{Sequence analysis}

Searching for sequence similarities revealed that the deduced amino acid sequence of $C l M y D 88$ shared higher identity with other known vertebrate MyD88s. According to the $C l M y D 88$ protein sequences of different species, it was found that $C l M y D 88$ shared the highest identity with MyD88 of Larimichthys crocea (GenBank accession No. ACL14361.1) (99\%; http://blast.ncbi.nlm.nih.gov/Blast.cgi). The alignment of MyD88 showed that some amino acid residues were highly conserved in different species (Figure 2), including Carassius carassius (AG057937.1), Danio rerio (NP-997979.2), Epinephelus coioides (ADZ53063.1), L. crocea (ACL14361.1), Lateolabrax japonicus (AEY83971.1), Miichthys miiuy (AFE85502.1), Mus musculus (ABG78744.1), Oplegnathus fasciatus (ADZ44623.1), Oryctolagus cuniculus (XP-002723915.1), Siniperca chuatsi (ADM25313.1), and Sus scrofa (ABW74617.1). In addition, an NJ-phylogenetic tree was generated using the complete MyD88 protein sequences deposited in NCBI (the sequence used was the same as that used for the MyD88 alignment described above) by MEGA 4.1 (Figure 3). Phylogenetic analysis indicated that ClMyD 88 shared higher identity in Sciaenidae such as L. crocea. This result is consistent with systematic evolution, indicating that the MyD88 gene could be a molecular index for systematic evolution.

\section{Predicted protein structure of $C l M y D 88$}

To further study the function of $C l M y D 88$ and its secondary structure, a 3D model was established using the PredicProtein software (https://www.predictprotein.org/) and the SWISS-MODEL long-distance server. The hierarchical neural network provided a structural 


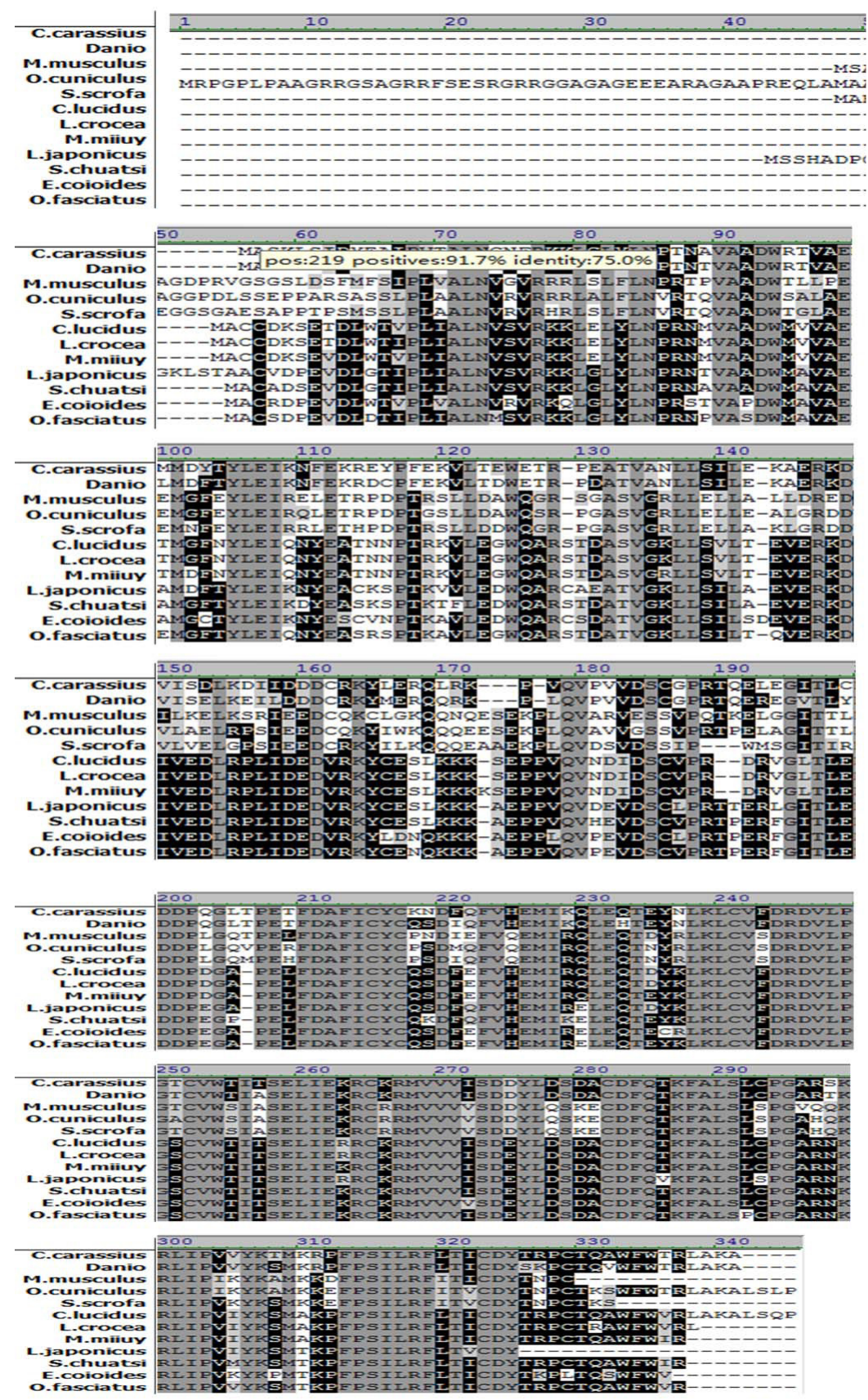

Figure 2. Alignment of the amino acid sequence of MyD88 with MyD88 sequences of other species. Gaps (-) were introduced to maximize the alignment. The relationships between residues are indicated as follows: non similar residues, black letters on a white background; conserved residues, black letters on a dark gray background; block of similarity, black letters on a light grey background; identical residues, white letters on a black background. 


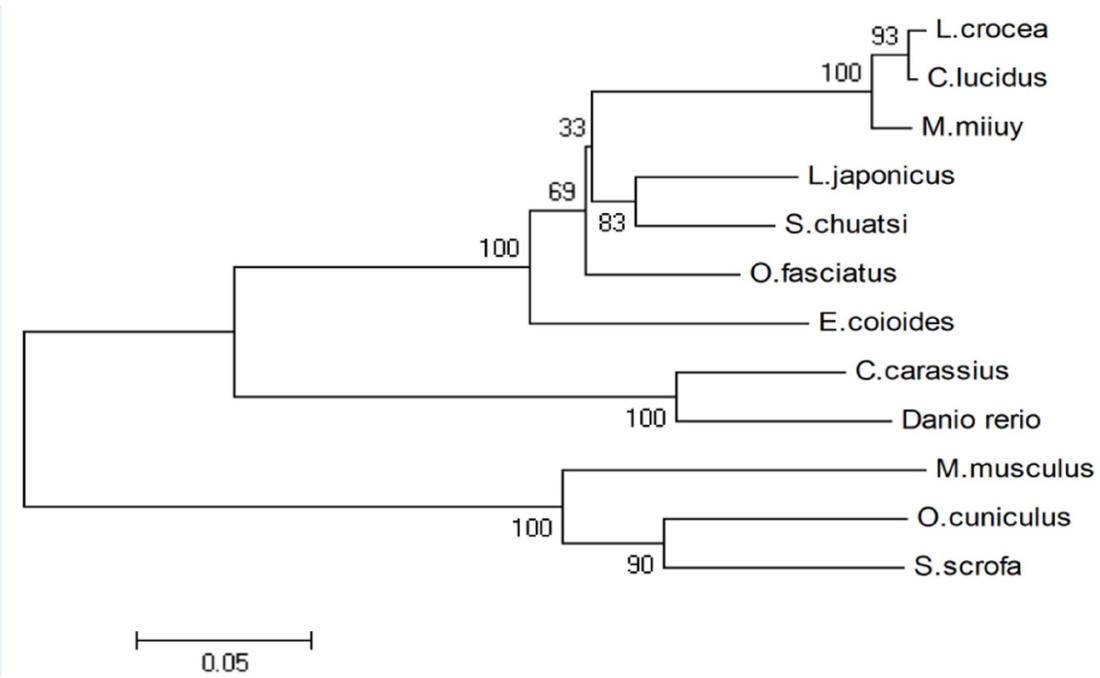

Figure 3. Evolutionary tree of MyD88. The tree is constructed by the neighbor-joining (NJ) algorithm using the MEGA 4.1 program based on multiple sequence alignment by Clustal W. The scale bar corresponds to estimated amino acid substitutions per site.

model of ClMyD 88 . This consisted of $44.3 \%$ alpha helices, $11.1 \%$ extended strands, and $44.6 \%$ random coils. This shows that alpha helices and random coils were the two major components in $C l M y D 88$. The deduced amino-acid sequences of $C l M y D 88$ were submitted to the SWISS-MODEL server. By searching the PDB data base with this program, a structure with 70\% identity to human MyD88 (PDB accession No. 2z5vA) was obtained. Comparative analysis revealed that although there is some difference in the MyD88 protein sequence, the spatial structure is a highly conserved cross species (Figure 4A and B).

\section{qRT-PCR analysis of ClMyDS8 in various tissues}

The mRNA expression pattern of a gene is closely related to its function. In this study, we examined the relative quantity of $C l M y D 88$ expression in several tissues from healthy $C$. lucidus by qRT-PCR (Figure 5). We found that $C l M y D 88$ was detectable in nearly all tissues and the expression levels in different tissues varied, with the highest level of $C l M y D 88$ expression in the gill followed by the hemocytes, and the weakest expression was observed in the brain and muscle. The MyD88 gene was broadly expressed in the blood, skin, dorsal muscle, gill, intestine, liver, spleen, heart, kidney, and brain of $P$. crocea, with the highest expression in the spleen and the weakest expression in the dorsal muscle (Yao et al., 2009). In Japanese flounders (Paralichthys olivaceus), the jfMyD88 transcript was strongly expressed in the gill, intestine, kidney, skin, and spleen; weakly expressed in the heart, liver, and stomach; and not measurable in the brain or muscle (Qiu et al., 2007). It was also reported that MyD88 of Japanese flounder was observed in the gill, spleen, and kidney, and that the number of MyD88-positive cells in the spleen increased after bacterial infection. These findings suggest that the level of MyD88 gene expression differs across species, probably because MyD88 acts in different ways in different tissues of these species. However, the spleen and gill may be two main sites that synthesize 

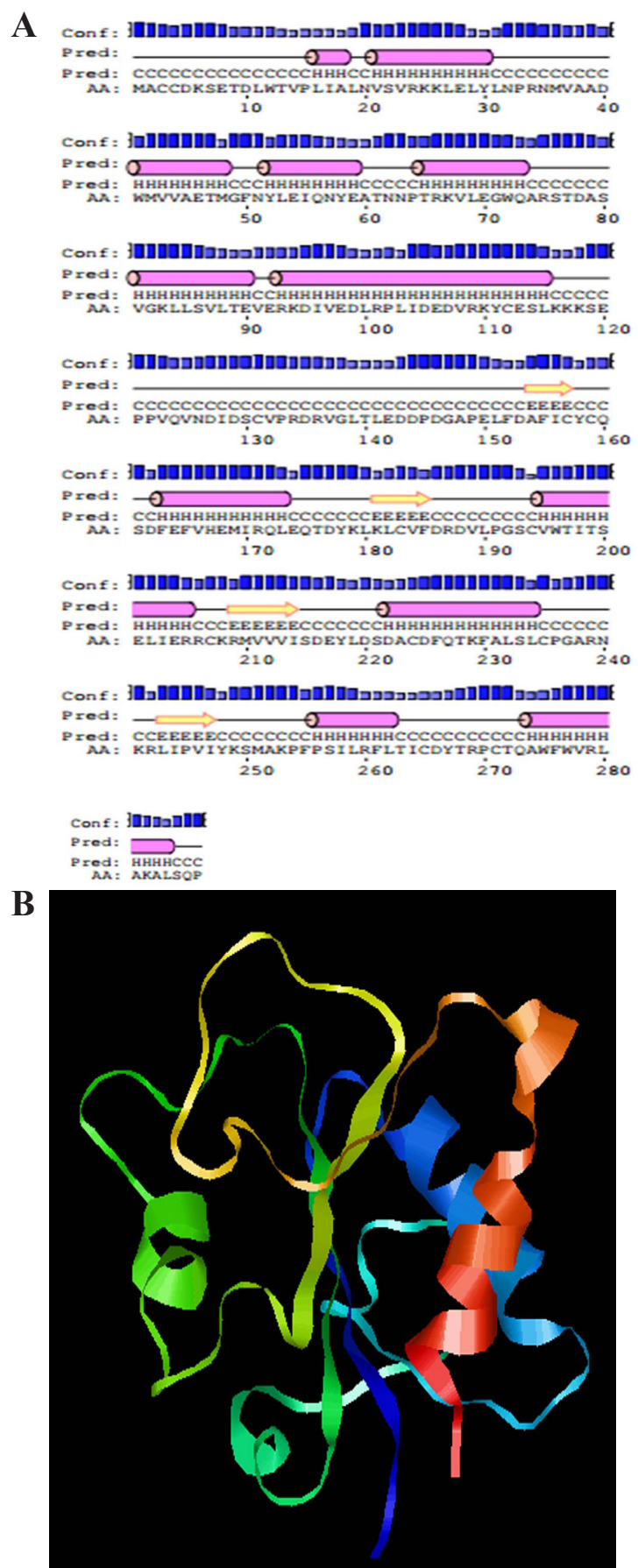

Figure 4. A. Secondary structure of the deduced $C l M y D 88$ polypeptide. The cylinder $(\mathrm{H})$ represents $\alpha$-helices, the arrow $(\mathrm{E})$ represents $\beta$-sheets, and the line (C) represents the random coil. B. Three-dimensional structure of the deduced $C l M y D 88$ polypeptide. 


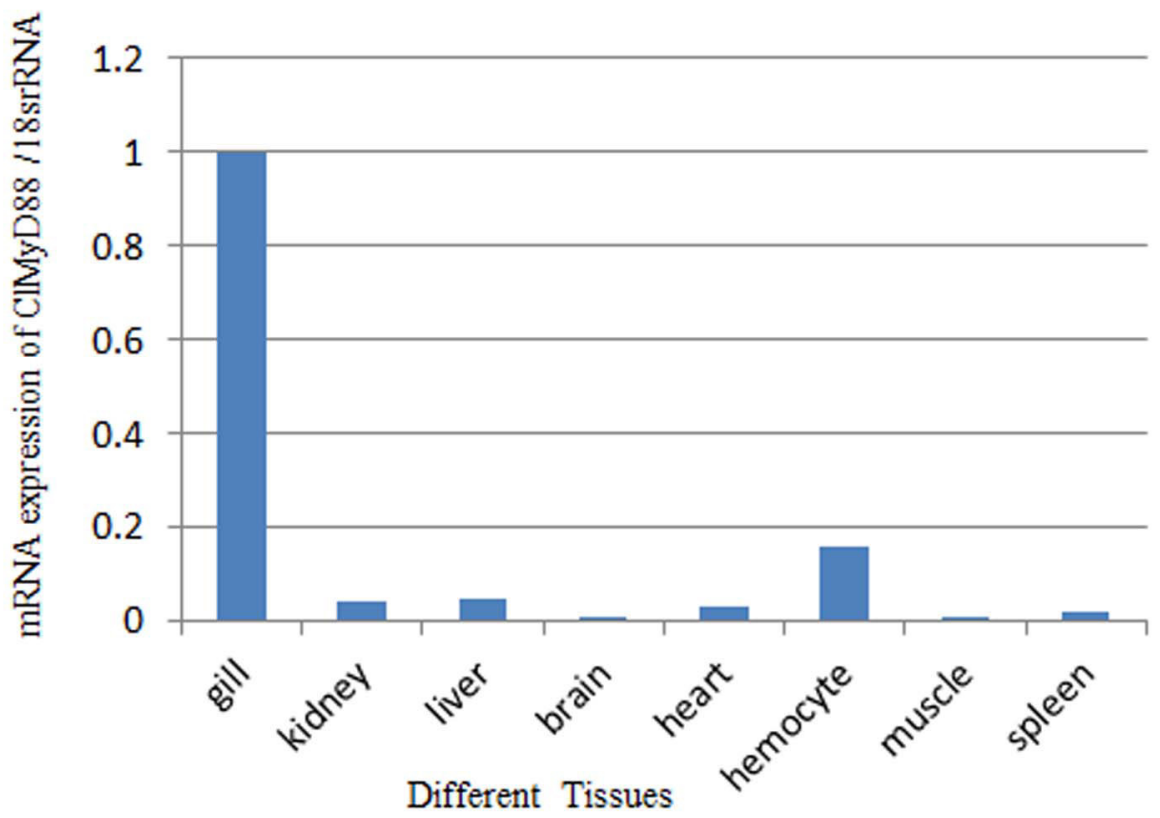

Figure 5. Relative expression of ClMyD88 in different tissues of Collichthys lucidus, including the gill, kidney, liver, brain, heart, hemocyte, muscle, and spleen. The amount of MyD88 mRNA was normalized to the 18S rRNA transcript level. Data are reported as means \pm SD of three repeated experiments. The "Y" axis represents the relative ratio of expression levels of MyD88/18S rRNA.

MyD88, and which play an important role in fish defense against bacterial infection.

\section{ACKNOWLEDGMENTS}

Research supported by the Basic Research Fund for State-level Nonprofit Research Institutes of ESCFRI, CAFS (\#Dong2012M06).

\section{REFERENCES}

Armant MA and Fenton MJ (2002). Toll-like receptors: a family of pattern-recognition receptors in mammals. Genome Biol. 3: 3011.

Beutler B (2005). The Toll-like receptors: analysis by forward genetic methods. Immunogenetics 57: 385-392.

Bilak H, Tauszig-Delamasure S and Imler JL (2003). Toll and Toll-like receptors in Drosophila. Biochem. Soc. Trans. 31: 648-651.

Bonizzi G and Karin M (2004). The two NF-kappaB activation pathways and their role in innate and adaptive immunity. Immunology 25: 280-288.

Burns K, Martinon F, Esslinger C and Pahl H (1998). MyD88, an adapter protein involved in interleukin-1 signaling. $J$. Biol. Chem. 273: 12203-12209.

Hawlisch H and Kohl J (2006). Complement and Toll-like receptors: Key regulators of adaptive immune responses. Mol. Immunol. 43: 13-21.

Itoh $\mathrm{N}$ and Nagata S (1993). A novel protein domain required for apoptosis. Mutational analysis of human Fas antigen. $J$. Biol. Chem. 268: 10932-10937.

Janssens S and Beyaert R (2002). A universal role for MyD88 in TLR/IL-1R-mediated signaling. Trends Biochem. Sci. 27: 474-482. 
Janssens S and Beyaert R (2003). Functional diversity and regulation of different interleukin-1 receptor-associated kinase (IRAK) family members. Mol. Cell 11: 293-302.

Jared CR, Glusman G, Rowen L and Kaur A (2005). The evolution of vertebrate Toll-like receptors. Proc. Natl. Acad. Sci. U.S. A. 102: 9577-9582.

Medzhitov R, Preston-Hurlburt P, Kopp E and Stadlen A (1998). MyD88 is an adaptor protein in the hToll/IL-1 receptor family signaling pathways. Mol. Cell 2: 253-258.

Muzio M, Ni J, Ping F and Dixit VM (1997). IRAK (Pelle) family member IRAK-2 and MyD88 as proximal mediators of IL-1 signaling. Science 278: 1612-1615.

Qian TL, Wang KR, Mu YN, Ao JQ and Chen X (2013). Molecular characterization and expression analysis of TLR 7 and TLR 8 homologs in large yellow croaker (Pseudosciaena crocea). Fish Shellfish Immunol. 35: 671-679.

Qiu LM, Song LS, Yu YD and Xu W (2007). Identification and characterization of a myeloid differentiation factor 88 (MyD88) cDNA from Zhikong scallop Chlamys farreri. Fish Shellfish Immunol. 23: 614-623.

Tauszig-Delamasure S, Bilak H, Capovilla M and Hoffmann JA (2002). Drosophila MyD88 is required for the response to fungal and Gram-positive bacterial infections. Nat. Immunol. 3: 91-97.

Takano T, Kondo H, Hirino T and Saito-Taki T (2006). Identification and characterization of a myeloid differentiation factor 88 (MyD88) cDNA and gene in Japanese flounder, Paralichthys olivaceus. Dev. Comp. Immunol. 30: 807-816.

Takeda K and Akira S (2005). Toll-like receptors in innate immunity. Int. Immunol. 17: 1-14.

Tartaglia LA and Ayres TM (1993). A novel domain within the $55 \mathrm{kd}$ TNF receptor signals cell death. Cell 74: 845.

Wang KR, Mu YN, Qian TL and Ao JQ (2013). Molecular characterization and expression analysis of Toll-like receptor 1 from large yellow croaker (Pseudosciaena crocea). Fish Shellfish Immunol. 35: 2046-2050.

Weber CH and Vincenz C (2001). The death domain superfamily: a tale of two interfaces. Trends Biochem. Sci. $26: 475$.

Wesche H, Henzel WJ, Shillinglaw W and Li S (1997). MyD88: An Adapter That Recruits IRAK to the IL-1 Receptor Complex. Immunity 7: 837-847.

Xiao X, Qin Q and Chen X (2011) Molecular characterization of a Toll-like receptor 22 homologue in large yellow croaker (Pseudosciaena crocea) and promoter activity analysis of its 5'-flanking sequence. Fish Shellfish Immunol. 30: 224-233.

Yao CL, Kong P, Wang ZY and Ji PF (2009). Molecular cloning and expression of MyD88 in large yellow croaker, Pseudosciaena crocea. Fish Shellfish Immunol. 26: 249-255. 